Article

\title{
A Framework for Stacked-Benefit Analysis of Distribution-Level Energy Storage Deployment
}

\author{
Anamika Dubey ${ }^{1, *}$, Pisitpol Chirapongsananurak ${ }^{2}$ and Surya Santoso ${ }^{3}$ \\ 1 School of Electrical Engineering and Computer Science, Washington State University, \\ Pullman, WA 99163, USA \\ 2 Department of Electrical Engineering, Chulalongkorn University, Bangkok 10330, Thailand; \\ pisitpol.c@chula.ac.th \\ 3 Department of Electrical and Computer Engineering, The University of Texas at Austin, \\ Austin, TX 78712, USA; ssantoso@mail.utexas.edu \\ * Correspondence: anamika.dubey@wsu.edu; Tel.: +1-509-335-1865
}

Academic Editor: Josep M. Guerrero

Received: 25 February 2017; Accepted: 23 March 2017; Published: 28 March 2017

\begin{abstract}
This paper presents a planning framework for integrating energy storage (ES) systems into the distribution system. An ES system is deployed to simultaneously provide multiple benefits, also known as stacked-benefits, for the feeder. The primary and secondary application scenarios for the feeder are identified. The proposed ES deployment approach includes the following steps: (1) size the ES system for primary application; (2) identify optimal ES locations based on both primary and secondary application scenarios; (3) calculate the ES accommodation capacity for each potential location; and (4) develop control methods for ES units and conduct grid impact analysis to demonstrate ES applications. For the selected feeder, the primary application for ES deployment is to provide the N-1 contingency requirement. During normal operating conditions, ES is programmed for multiple secondary applications: voltage management and ancillary services by frequency regulation. A probabilistic approach is presented to obtain the optimal ES size for providing the N-1 contingency requirement. Optimal ES locations are obtained based on secondary application scenarios. Real and reactive power control methods are developed to demonstrate the viability of deploying an ES system for simultaneously providing multiple applications. The simulation results show that ES can successfully provide the stacked-benefits for the distribution circuit. The proposed framework is generic and can be employed for the ES integration analysis of any feeder, with different sets of primary and secondary applications.
\end{abstract}

Keywords: smart grid; power distribution system; distributed energy resources (DERs); energy storage (ES); photovoltaic system (PV)

\section{Introduction}

With the advances in smart-grid technologies and the integration of distributed energy resources (DERs), the electric distribution system has been changing rapidly [1-5]. These infrastructural changes pose multiple challenges for electric distribution service providers. The inherent variability of renewable generation and the vulnerability of traditional power systems to the demand and load stochasticity, can potentially result in poor service reliability and quality [6-9]. Energy storage (ES) systems have been identified as a potential solution to maintain strict power quality and reliability standards, by both utilities and researchers [10-12]. Including ES technology in distribution plans can enable utilities, system operators, and end users to increase power reliability and reduce the cost of electricity [12-14]. Additionally, ES systems aid distribution grid flexibility, as they can help integrate variable generation resources such as wind and solar [15-18]. Given various applications of ES systems, 
a distribution system planning framework is called for that incorporates the potential impacts and benefits of ES deployments.

Several studies have assessed the values of integrating ES systems into the distribution circuits. To reduce the impacts of renewable energy resources, ES has been used for multiple grid support applications, such as load management, voltage regulation, and frequency support [15-18]. References [18-20] describe the benefit of ES in providing a photovoltaic (PV) smoothing service by reducing the PV generation variability. ES can also be employed to provide peak load shaving [21] and to support the grid during outages [22]. According to [18,21,23], ES is efficient at providing voltage regulation in distribution circuits with a high penetration of PV. The application of ES in providing frequency regulation is presented in $[18,24,25]$. So far, however, these technical studies have not quantified the benefits of ES deployment in simultaneously providing multiple grid benefits. With the ongoing efforts to make ES systems cost-competitive [26] and an increasing number of successful ES demonstration studies [27], a systematic approach to evaluate the grid impacts and benefits of ES systems is called for. Grid integration tools should be able to identify suitable ES applications, sizes, and deployment locations, by using domain-specific knowledge.

The objective of this study is to develop a systematic approach for integrating an ES system into a given distribution grid. This paper presents an approach to deploy an ES system, to provide multiple grid benefits, also termed stacked-benefits, for the feeder. The optimal ES size and locations are obtained based on the stacked-benefit application scenario and distribution system grid impact analysis. This paper also shows that ES can simultaneously provide multiple benefits for the distribution feeder. For this study, the ES system is expected to provide the following stacked benefits: (1) primary application- $\mathrm{N}-1$ contingency requirements; and (2) secondary applications-voltage management and frequency support applications. Note that, although the proposed framework is applicable to any ES technology capable of providing active and reactive power support, the presented framework mostly focusses on battery ES systems.

\subsection{Distribution-Level Energy Storage Deployment}

A brief review of use cases for distribution-level ES deployment is presented in this section. Broadly, ES systems can be deployed to provide system-level benefits and customer-level benefits. When deployed for system-level benefits, ES systems are programmed to provide operational benefits to utility companies in the form of grid services. When deployed for customer-level benefits, ES systems are programmed to meet specific service quality and reliability requirements for the individual customers.

\subsubsection{System-Level Benefits}

- Defer Distribution System Upgrades-With an increase in the yearly peak load demand, the substation and distribution lines may require expensive upgrades. If the cost-benefits analysis allows, an ES system may be deployed to decrease the peak load demand and defer any immediate distribution system upgrades.

- Support DER Integration-As stated previously, integrating a large percentage of DERs can result in multiple grid issues requiring grid upgrades. ES has been shown to be beneficial in mitigating the impacts of DERs by supporting applications such as load shifting to mitigate off-peak generation, voltage management to mitigate overvoltage concerns, and voltage control to mitigate DER variability.

- Support Energy Arbitrage/Electricity Price Shift-ES can help distribution utilities in decreasing the yearly cost of the electricity by participating in the electricity market. ES can be programmed to charge and discharge as the electricity rates fluctuate, thus decreasing yearly electricity costs.

- Provide Ancillary Services-ES can be programmed to provide ancillary services for frequency regulation based on the feeder's open access transmission tariff. 


\subsubsection{Customer-Level Benefits}

- Voltage Management-Traditionally, voltage management is pursued using load-tap changers and capacitor banks. For a feeder with high DER penetrations, the voltage management will require new methods using faster voltage control devices. In such cases, ES inverters can provide voltage regulation services to mitigate overvoltage and voltage variation concerns.

- Provide Reliability Benefits-When deployed for providing reliability benefits, ES will discharge to support the connected loads during an outage, thus decreasing both the Customer Average Interruption Frequency Index (CAIFI) and the Customer Average Interruption Duration Index (CAIDI).

- Demand Charge Management-The demand charge is a billing mechanism used to recover the cost of providing a transmission and distribution service to commercial customers. The demand charge for a month is calculated based on the largest peak demand recorded over the month. ES systems are deployed to decrease the monthly peak load demand and decrease the total customer demand charge.

\subsection{Objectives and Assumptions}

The objective of this paper is to present a systematic approach for planning the deployment of ES units for a distribution system. For the deployment to be economical, ES should be integrated to provide multiple grid benefits, also known as stacked benefits. Depending upon the feeder, the planner first needs to identify the primary or secondary benefits of ES deployment. The primary application is the one that is either most critical or will generate the most revenue for the distribution system. The secondary applications correspond to the additional grid benefits that ES can provide, in addition to the primary application. Multiple secondary benefits of ES should be identified, depending upon the feeder and the use case. Next, a systematic approach should be developed to evaluate the efficacy of ES deployment in providing multiple/stacked grid services.

In this paper, we present a step-by-step approach for ES integration analysis, starting with the assessment of ES requirements for the grid, the method used to calculate the ES system size, the identification of optimal ES locations while accounting for the grid impacts of ES deployment, and finally, the verification of ES benefits through simulation. We identify three important aspects of ES deployment: (1) ES deployment should be economical; (2) ES deployment should not result in any operational concern for the grid; and (3) ES must provide the intended grid benefits. In this regard, we demonstrate a stacked application case for ES deployment. The ES is sized for the primary application scenario. To ensure that ES deployment does not cause additional operational concerns, the largest allowable limits of the ES capacity for each potential ES location are determined. Next, a grid impact analysis is completed to verify and demonstrate that an ES can provide the intended grid benefits.

In this study, the ES system is sized and deployed for the following stacked benefit application scenario: (1) Primary application-provide N-1 contingency for the substation transformer; and (2) Secondary applications-frequency regulation and voltage management services. The distribution circuit selected for this analysis is adapted from EPRI's 34.5-kV test circuit [26]. We also include the data and modeling requirement to enable the proposed ES integration analysis framework (see Section 2). The proposed framework for ES integration analysis is detailed in Section 3, followed by the verification method and results in Section 4. Finally, conclusions are presented Section 5.

\subsection{Contributions}

This paper provides a systematic approach for evaluating the grid impacts and benefits of ES deployment for a multiple/stacked application scenario. The ES system is planned to simultaneously provide multiple grid benefits. A primary grid benefit, potentially the most critical application, is identified. The ES is programmed to provide secondary benefits, when not required for the primary application. We develop a control method to program the ES system for voltage management 
and frequency regulation support. Voltage management is achieved using Volt-VAR control, while frequency regulation is provided by charging and discharging the ES, based on the frequency control signal. We also present an approach to size the ES, based on the primary benefit i.e., N-1 contingency requirement using a percentile analysis. Next, optimal locations for ES systems are identified, while including their impacts on the feeder. Finally, using simulations, we demonstrate the proposed approach for the selected feeder.

The purpose of this paper is to provide a methodology that can essentially help include ES into the distribution planning framework. In this regard, the proposed approach is generic and applicable to a different set of primary and secondary application cases. We also provide details on the data and model requirements for the proposed study. Therefore, the proposed framework can be easily used by utility providers to assess the applicability of ES deployment for their feeder.

\section{Analysis Tools and Test Circuit}

The analysis tools and test circuit used for the study are detailed in this section. We simulate the proposed ES integration framework using OpenDSS [28] and MATLAB. The analysis is conducted using a test circuit adapted from a 34.5-kV EPRI test feeder [29] available from an open-source for distribution system analysis.

\subsection{Analysis Tools}

The proposed application framework requires a detailed distribution system feeder model and an interface to simulate and analyze multiple case studies. This paper uses OpenDSS, an open-source distribution system simulator, to simulate the distribution circuit model [28]. We have used MATLAB as an interface to OpenDSS, to conveniently simulate multiple case studies, and project load and generation scenarios. A detailed feeder model starting from the substation down to individual customer locations is simulated in OpenDSS. Additionally, representative models for customer loads are also developed using the substation load data and customer load characteristics. The ES units are modeled using the equipment model for ES available in OpenDSS.

\subsubsection{Distribution Circuit Simulator-OpenDSS}

OpenDSS [28] is a comprehensive electrical system simulation tool for electric utility distribution systems, supporting all frequency domain analyses. It supports the quasi-static unbalanced three-phase power flow analysis for radial and looped networks, while including user-defined control algorithms for shunt capacitors, voltage regulators, on-load tap changers, and smart-inverters. The simulator also facilitates DER integration analysis and provides pre-defined models of DERs and their control blocks. OpenDSS can be implemented as both a stand-alone executable program and an in-process Component Object Model (COM) server DLL, designed to be driven by a variety of existing software platforms. In this study, OpenDSS is executed using the MATLAB program.

\subsubsection{Energy Storage Model}

The storage element is modeled as a generator and is dispatched to either generate power by discharging, or consume power by charging, within its power rating and its stored energy capacity. In OpenDSS, ES is modeled as a generator (Figure 1). The storage element can also produce or absorb reactive power within the $\mathrm{kVA}$ rating of its inverter. The ES model supports power flow analysis in multiple time-scales, including Snapshot, Daily, Yearly, and Duty cycle modes [28]. In addition to equipment model and solution interfaces, OpenDSS also provides several control models for ES systems. The control modes are required for simulating the cases to demonstrate that the utility of ES is providing grid benefits. 


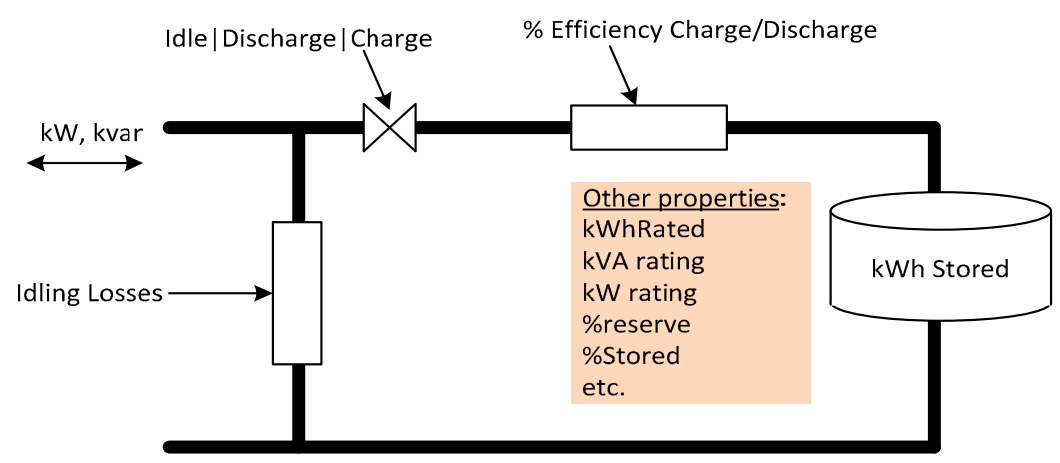

Figure 1. Simplified block diagram of OpenDSS model for an ES system [28].

\subsubsection{Required Circuit Data}

The data requirement for the proposed analysis framework is detailed in this section. Note that a detailed feeder model, including circuit data, load data, and DER data, is required to conduct a realistic distribution planning analysis (see Table 1). There are three categories of required data:

- Circuit data-Includes the one-line diagram of the feeder, substation model, distribution line characteristics including impedance, ampacity, voltage levels, and connections, and details regarding distribution transformers and capacitor banks.

- Load data-Includes the hourly load demand data measured at the substation for one year or more, as well as the characteristics of individual customer loads distributed across the feeder.

- DER data-Details of existing and future DER integration is required for the planning analysis. For example, for existing PV systems, necessary data include PV panels' location, size, and characteristics. Additionally, high-resolution data (typically 1 s) on PV generation for an entire year and a few selected representative days should be provided.

Table 1. Required circuit data for ES integration analysis.

\begin{tabular}{|c|c|}
\hline \multicolumn{2}{|r|}{ Circuit Data } \\
\hline Source & $\begin{array}{l}\text { Line-line voltage }(\mathrm{kV}) \text { at the source bus, short-circuit MVA at the high-side bus for each } \\
\text { substation (three-phase and line-ground) }\end{array}$ \\
\hline Substation & $\begin{array}{l}\text { Transformer connections, high- and low-side voltages, transformer size, } X / R \text { ratio, and } \\
\text { leakage impedance (\% on substation MVA), substation bus configuration and the number } \\
\text { of feeders served from each bus, substation LTC or regulator settings }\end{array}$ \\
\hline Distribution lines & $\begin{array}{l}\text { Bus connections, conductor size, type, length, construction, emergency ampacity, phase } \\
\text { and sequence impedance }\end{array}$ \\
\hline Distribution transformer & $\begin{array}{l}\text { Primary and secondary buses connected to the transformer, transformer MVA rating, } \\
\text { high—and low-side voltages, size (kVA), X/R ratio, leakage impedance }\end{array}$ \\
\hline Capacitor banks & $\begin{array}{l}\text { Bus connection and connection type, size, control mechanism (fixed, voltage, current, kvar, } \\
\text { time, etc.), and control settings (on/off settings) }\end{array}$ \\
\hline \multicolumn{2}{|r|}{ Load Data } \\
\hline Metered Load Data at Substation & $\begin{array}{l}\text { 1-year data with appropriate granularity (at least in hourly intervals) of real energy (MWh) } \\
\text { and reactive energy (Mvarh) into and out of the substation, real (MW) and reactive (Mvar) } \\
\text { power demand at the substation. }\end{array}$ \\
\hline Customer Loads & $\begin{array}{l}\text { Rated load demand at individual customer locations (kW, power factor, allocation factor), } \\
\text { load duration curves (15-min interval) aggregated for the circuit and for individual } \\
\text { customers', if available. }\end{array}$ \\
\hline \multicolumn{2}{|r|}{ DER Data } \\
\hline Existing DERs and generation profiles & $\begin{array}{l}\text { Existing DER locations and size along the feeder and DER control mechanism. DER } \\
\text { generation data provided in } 1 \mathrm{sec} \text { resolution - for the entire year or longer (if available) or } \\
\text { generation profiles representing typical days, for example, a clear day, mild, moderate, and } \\
\text { high variability, and an overcast day should be provided. }\end{array}$ \\
\hline
\end{tabular}




\subsection{Selected Distribution Circuit}

The one-line diagram for the test circuit is shown in Figure 2. In this study, we simulate two identical substation transformers supplying two distribution feeders with identical load demand characteristics and peak load demands. Note that under the N-1 contingency case, one of the transformers is out-of-service and the other transformer supplies both feeders. The distribution feeder supplied by Transformer T1, i.e., Feeder 1, is adapted from the 34.5-kV EPRI test feeder [29]. The feeder supplied by Transformer T2 is represented as an equivalent load with an identical load profile and load demand characteristics as Feeder 1. Both substation transformers are rated as having a normal MVA rating of 45 MVA and emergency rating of 75 MVA. The three-phase short-circuit capacity at the secondary of either substation transformer is 422 MVA.

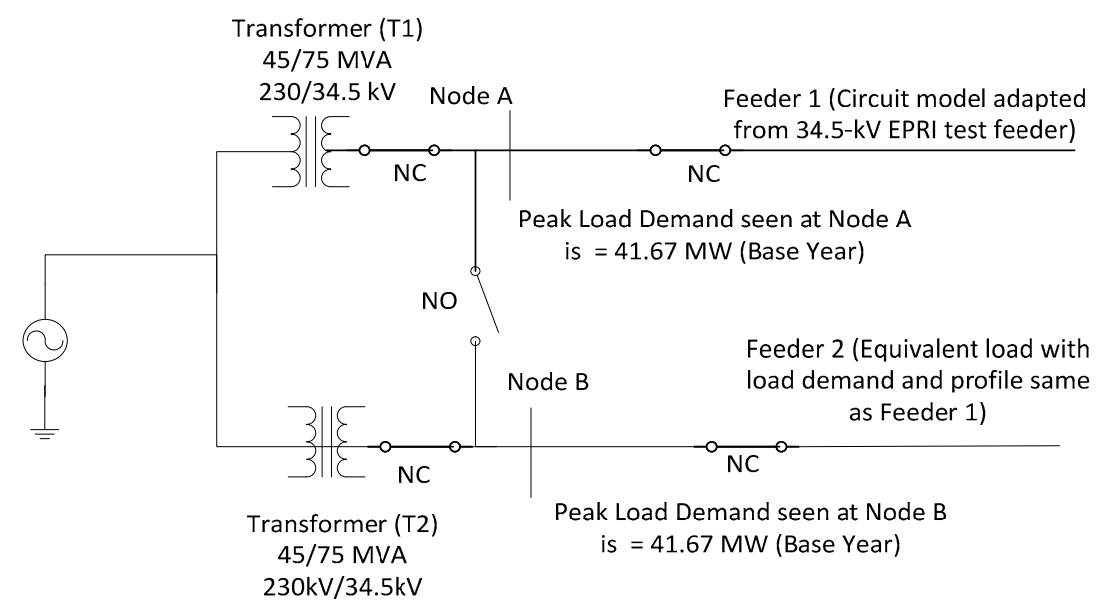

Figure 2. One line diagram of the substation configuration.

The one-line diagram of Feeder 1 is shown in Figure 3 [30]. In Figure 3, the colors represent the distance of the feeder from the substation. The distance (in $\mathrm{km}$ ) associated with each color is also shown in the figure. The detailed circuit characteristics for Feeder 1, including bus connections, primary-feeder characteristics, service transformer data, secondary-feeder characteristics, and customer load data, are available. Also, the yearly load profile measured at the substation transformer is available for one year, at a 1-h resolution. This circuit has three capacitors of 0.9, 1.2 and 1.2 Mvar. These capacitors are fixed and always online. The substation transformer is equipped with a load tap changer (LTC). Five PV systems are installed at a $34.5 \mathrm{kV}$ voltage level of Feeder 1. Each PV is connected to the feeder via a 1.2-MVA 34.5/0.48-kV service transformer and has a capacity of $1 \mathrm{MW}$, with a total installed capacity of $5 \mathrm{MW}$.

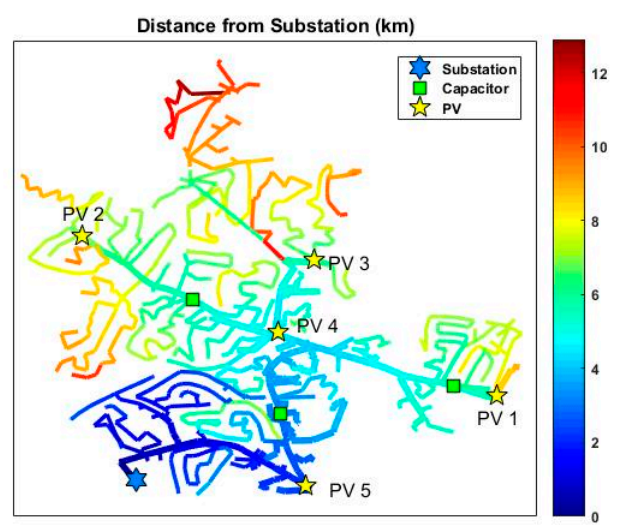

Figure 3. Detailed one-line diagram for Feeder 1. 
Using the available load demand data and circuit characteristics, a power flow analysis is conducted for one year. The load demand recorded at the secondary of one the substation transformers for the given year, is shown in Figure 4. Note that the recorded load demand does not take PV generation into account. The peak load demand when the existing PV systems are not generating for the given year is equal to 41.67 MVA. The peak load demand without PV generation is reported, because the PV is a variable and uncertain resource and should not be included for contingency and peak load management analysis. The PV profile and impacts will be included when studying voltage support and frequency regulation applications. In this study, for the primary application i.e., to meet N-1 contingency criteria, ES is planned for a duration of five years, starting from the base year (Year 1). A load growth rate of $1 \%$ per year is assumed to project the yearly load demand for the next five years.

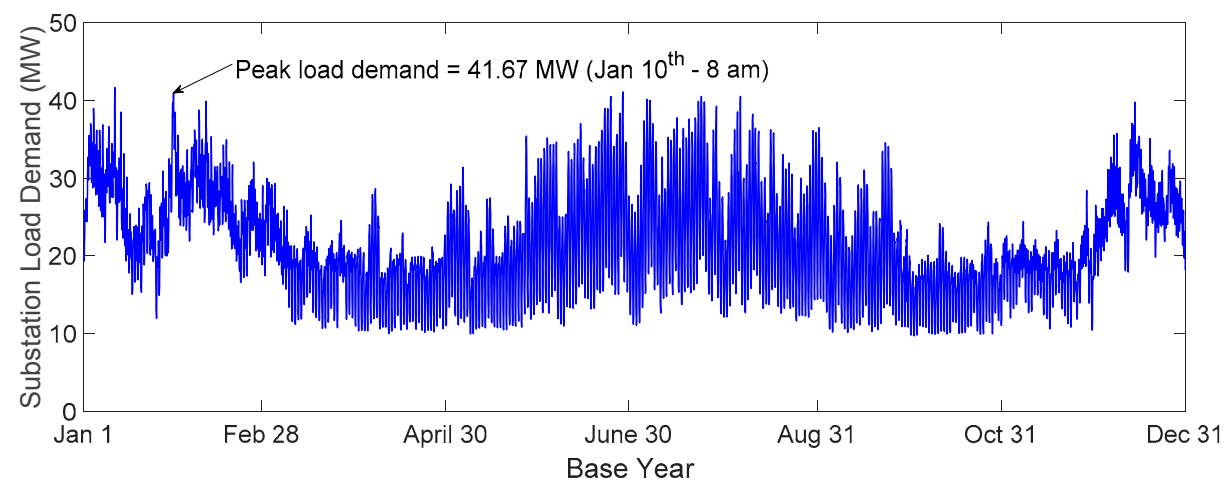

Figure 4. Yearly load demand recorded at the secondary of one of the substation transformers for the base year-Year 1 (PV systems are assumed to be not generating).

\section{Proposed Analysis Framework}

In this paper, we present a planning approach for the stacked benefits application of an ES simultaneously providing: N-1 contingency, voltage management, and frequency regulation services. The primary application for the ES is providing N-1 contingency support. Therefore, when the feeder requires contingency support, $100 \%$ of the ES is deployed to mitigate the substation transformer overloading. When contingency support is not required, ES is committed to providing secondary benefits, i.e., voltage support and frequency regulation. The energy storage commitment for both voltage management and frequency regulation support is assumed to be $50 \%$.

\subsection{Define/Identify Application Scenario}

In this section, the details of both primary and secondary application cases are presented, along with an ES control scheme used to provide the stacked grid benefits.

\subsubsection{Primary Application Scenario-N-1 Contingency}

The ES system is primarily deployed to provide the N-1 contingency requirement for the substation transformer. The one-line diagram for the distribution circuit under analysis is shown in Figure 2. Two identical transformers with a nominal rating of 45 MVA and an emergency rating of 75 MVA are connected to the substation. Each transformer serves a distribution feeder with a peak load demand of 41.67 MW, measured during the base year (Year 1). It is assumed that, under normal conditions, both transformers are in service. The N-1 contingency condition is simulated by applying a fault at the secondary of the transformer T1. In this case, the other transformer, T2, is required to serve both feeders (see Figure 5). Based on the peak load demands of both feeders, the worst-case demand of transformer T2 during the base year is $83.34 \mathrm{MW}$. The $\mathrm{N}-1$ contingency requirement is not satisfied for the base year, as the worst-case load demand exceeds the transformer's emergency rating. 


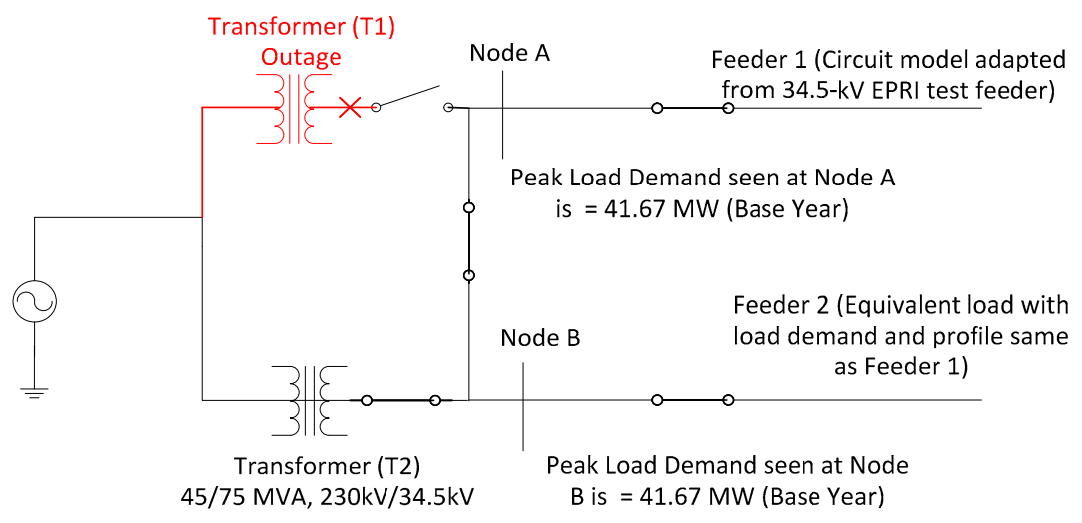

Figure 5. Simulated N-1 contingency case.

The following assumptions are made for ES deployment to provide N-1 contingency support:

1. The ES is planned for five years, since the N-1 contingency requirement is not met.

2. The yearly load profile at a 1-h resolution is available for the base year. A $1 \%$ load growth per year is assumed to project the yearly load demand for the future years.

3. The ES system size is determined based on a percentile analysis on the five-year load demand data, including available measurements for the base year and projected measurements for the future years.

\subsubsection{Secondary Application Scenarios-Voltage Management and Frequency Regulation}

As mentioned before, the ES in this study is deployed to provide the following secondary benefits: (1) voltage management; and (2) frequency regulation or ancillary services by participating in the ISO market. An ES participates in ancillary service/frequency regulation by generating or absorbing real power based on the frequency regulation signal [31]. Voltage management is achieved using Volt-VAR control of the smart inverter connected to the ES system. Since the feeder contains PV systems, the ES is deployed to mitigate voltage variations resulting from PV variability. Note that the feeder voltages will also fluctuate due to the participation of the ES system in providing ancillary services. Thus, ES needs to mitigate the voltage variation due to both PV variability and the ES charge/discharge frequency regulation schedule. The ES system is committed to providing $50 \%$ to voltage support and another $50 \%$ to the frequency regulation service.

Voltage Management

For the voltage management service, the control objective is to mitigate the voltage variations due to both PV variability and the ES charge/discharge frequency regulation schedule. The feeder voltages can be regulated using real and reactive power control, although a reactive power control mode may incur an additional cost for the smart inverter. Additionally, this control method, unlike real power control, does not deteriorate the battery life-cycle. Also, when it is in reactive power control mode, ES is not generating or absorbing any real power, so it need not be oversized to meet multiple objectives.

In this paper, the reactive power control mode is implemented for voltage management. The inverter of ES is controlled to generate or absorb the reactive power, to mitigate the voltage variations due to both PV variability and the ES charge/discharge frequency regulation schedule. The reactive power supplied or consumed by the ES inverter is calculated from both the change in PV generation and the change in ES charge/discharge (1).

$$
\Delta Q_{E S}=\left(\Delta P_{P V}+\Delta P_{E S}\right) \times \frac{R_{t h}}{X_{t h}}
$$

where, $\Delta Q_{E S}$ is the change in the reactive power supplied or absorbed by the ES inverter, $\Delta P_{P V}$ is the change in the PV generation power, $\Delta P_{E S}$ is the change in the ES charge or discharge power in 
response to the frequency regulation schedule, $R_{t h}$ is the real part of the short-circuit impedance at the ES location, and $X_{t h}$ is the imaginary part of the short-circuit impedance at the ES location. Note that $\Delta P_{P V}$ and $\Delta P_{E S}$ are measured as the difference between the present PV and ES real power, and the 15- and 30-min moving average PV and ES real power, respectively. The PV generation follows the solar irradiance profile curve recorded on a high variability day with a one-second resolution, shown in Figure 6a.

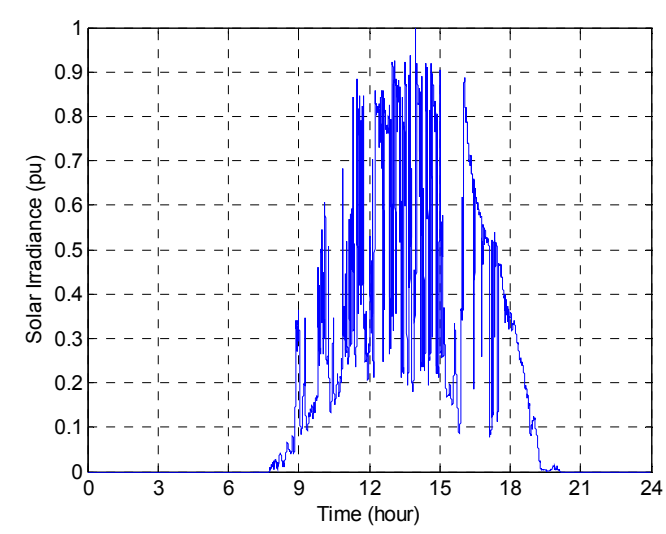

(a)

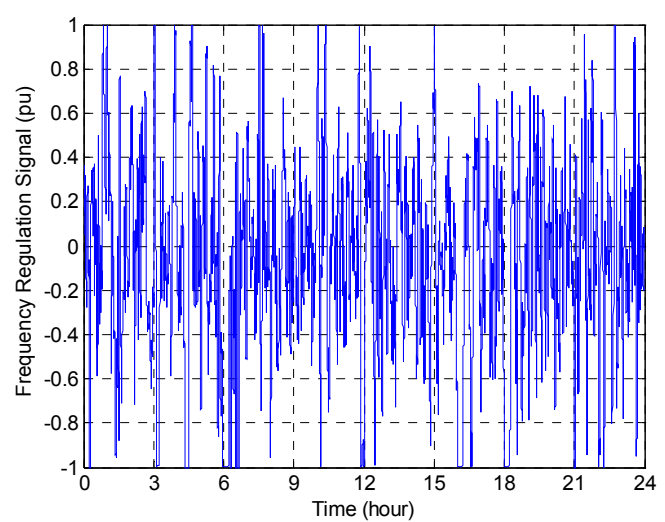

(b)

Figure 6. Assumptions for the analysis: (a) PV generation profile; (b) Frequency regulation signal.

Frequency Regulation

For the frequency regulation service, the control objective is to consume or produce real power to participate in ancillary service/frequency regulation in an ISO market. In this paper, ES is programmed to charge or discharge, according to the frequency regulation signal provided by [32]. There are two regulation signals calculated from the area control error signal (ACE): RegA and RegD. Both RegA and RegD signals are in per-unit with 2-s resolution.

- RegA Signal: This signal is generated by passing the ACE signal through a low pass filter. The RegA signal is used for traditional regulating resources.

- RegD Signal: This signal is generated by passing the ACE signal through a high pass filter. The RegD signal is used for dynamic or fast response resources.

This paper uses a 2-s resolution $\operatorname{Reg} \mathrm{D}$ frequency regulation signal generated for one day, as shown in Figure 6b [32]. For the frequency regulation service, the positive value signal instructs the ES to discharge (acting as a generator), while the negative signal instructs the ES to charge (acting as a load). The signal (in per unit) is multiplied by the power rating of the ES and the commitment level $(50 \%)$, to obtain the ES charge and discharge schedule (in MW). Note that the ES schedule is also of a 2-sec resolution. The ES charge and discharge schedule fluctuates over the simulation period. This fluctuation results in variations in feeder voltages.

\subsection{Energy Storage Deployment Methodology}

The proposed ES deployment method is detailed in this section. First, the energy storage size is determined based on the N-1 contingency criteria. It is assumed that ES is deployed to provide $\mathrm{N}-1$ contingency benefits for five years. A statistical analysis is completed for the present and projected load demand data. A $1 \%$ load growth is assumed. Based on the statistical analysis, the typical substation load demand and overload duration are obtained, which are used to calculate the ES size. Next, the best locations for deploying ES along the feeder are determined. Note that a few grid applications, for example, voltage management and reliability services, are sensitive to the ES deployment locations, while others, including frequency regulation and peak-load shaving, are not. Therefore, in this paper, 
ES systems are collocated with PV systems for feeder voltage management, to mitigate the impacts of a variable PV generation profile. Since ES deployment should not result in any additional integrational challenges, the ES accommodation limit is calculated at each PV location. Based on the accommodation limit, ES is sized for each location. Finally, a grid impact analysis is simulated to verify and demonstrate that ES is successfully able to provide the intended grid benefits.

\subsubsection{Calculate Energy Storage Size}

ES needs to be sized for the power subsystem (MW) and energy subsystem (MWh) ratings. The power subsystem rating defines the largest capacity that ES can supply. The energy subsystem rating defines the total energy that an ES can provide without recharging. ES is sized to meet the N-1 contingency requirement for a duration of five years, including the base year, for the selected distribution system. The N-1 contingency requirement is satisfied if the transformer can supply both feeders without recording any overloading in an emergency situation i.e., when the other transformer is out-of-service. Given the uncertainty in the load demand, sizing ES for the worst case peak load demand recorded over five years is not advisable. In this study, the ES size is determined using a statistical analysis on five-year substation load demand data under an N-1 contingency scenario. The statistically representative peak load demand and energy supplied above the transformer's rating are obtained using a percentile analysis on the base and projected year load demand data. The energy storage is sized for the typical peak load (MW) and the typical energy supplied above the transformer's emergency rating (MWh).

\section{Percentile Analysis on Load Data}

The yearly load demand is available for the base year at an hourly interval. The base year load demand is projected for the next four years, assuming a load growth of $1 \%$ per year. This results in a total of 43,800 data points, with 8760 each year. A percentile analysis is completed for the obtained data set. Figure 7 shows the percentile plot for the five-year load demand data. Here, the load demand corresponding to the $x$ percentile, say $P_{x}$, means that $x \%$ of hourly load demands will fall below $P_{x}$. By this definition, $98 \%$ of the load demand, recorded and projected over five-years, will fall below the 98-percentile load demand value.

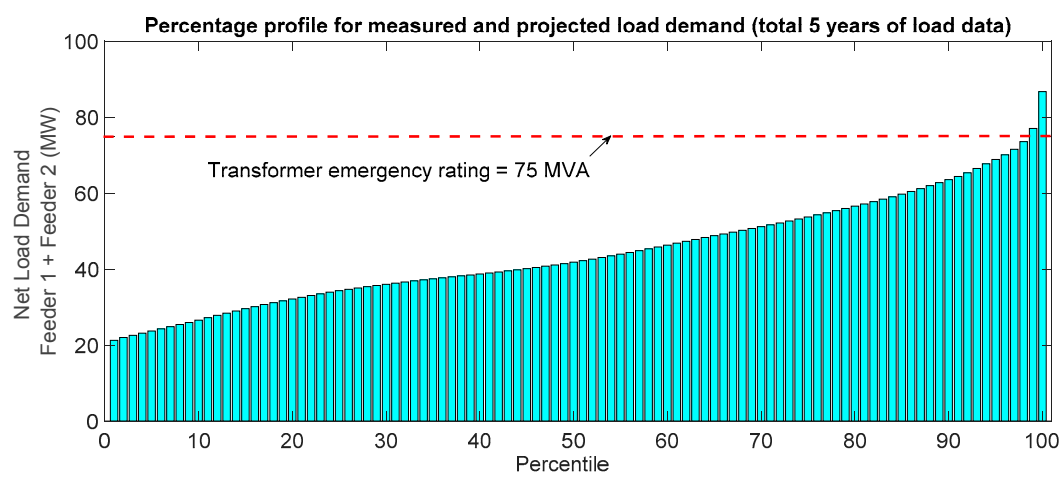

Figure 7. Percentage profile obtained for the five-year load data.

Since ES needs to be sized for the cases of substation overloading, a percentile analysis is only completed for those hours when the load demand is greater than the substation transformer rating (see Figure 8). In Figure 8, the load demand $\left(P_{x}\right)$ corresponding to the $x$-percentile denotes that, if a transformer overloading is recorded, it will be less than $P_{x}$, with a $x \%$ probability. Therefore, the load demand corresponding to the 95 th-percentile $\left(P_{95}=82.99 \mathrm{MW}\right)$ indicates that the transformer overloading recorded (in five years) will be less than $82.99 \mathrm{MW}$, with a 0.95 probability. Note that the representative peak load demand decreases significantly in the 75th-percentile, to $80.74 \mathrm{MW}$. 


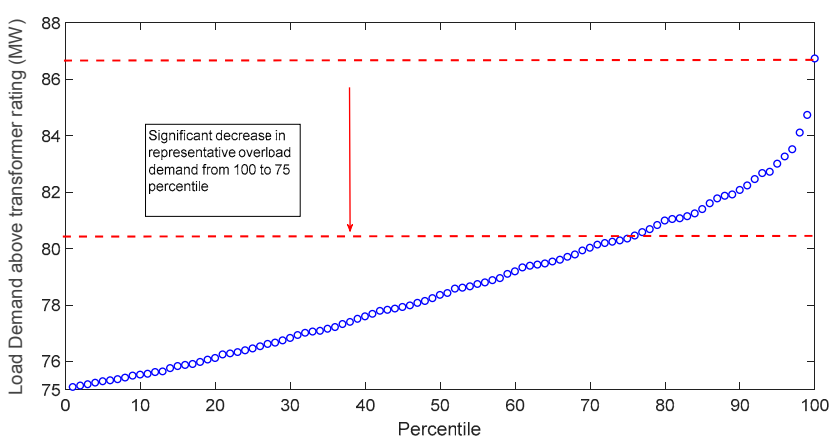

\begin{tabular}{cc}
\hline Percentile & $\begin{array}{c}\text { Peak Load } \\
\text { (MW) }\end{array}$ \\
\hline 100 & 86.72 \\
99 & 84.72 \\
97 & 83.50 \\
95 & 82.99 \\
90 & 82.07 \\
75 & 80.74 \\
50 (median) & 78.35 \\
\hline
\end{tabular}

Figure 8. Percentile profile for the overload demand (demand $>75 \mathrm{MW}$ ) and selected percentile values.

A similar analysis is conducted for the MWh served above the transformer's emergency rating (75 MVA) over the span of five years. The percentile plot and representative percentile MWh above the transformer rating are shown in Figure 9. The 75th-percentile value for the MWh served above the transformer rating implies that, under N-1 contingency, the transformer will be serving an overload energy less than or equal to $30.28 \mathrm{MWh}$, with a probability of 0.75 in the five-year planning period.

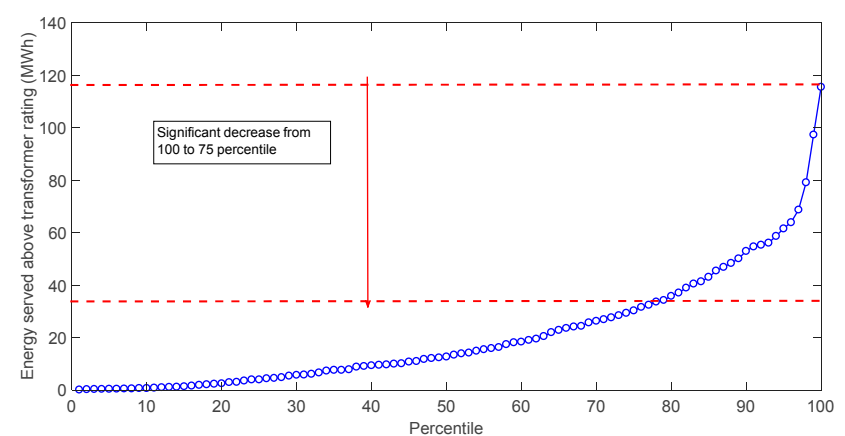

\begin{tabular}{cc}
\hline Percentile & $\begin{array}{c}\text { MWh Served above } \\
\text { Transformer Rating }\end{array}$ \\
\hline 100 & 115.53 \\
99 & 97.34 \\
97 & 68.73 \\
95 & 61.56 \\
90 & 52.98 \\
75 & 30.28 \\
50 & 12.71 \\
\hline
\end{tabular}

Figure 9. Percentile profile for the energy demand above the transformer rating and selected percentile values.

\section{Energy Storage Size}

The ES size for both the MW and MWh ratings are determined based on the results obtained from the percentile analysis of the five-year load demand data. For the worst-case scenario and to avoid any capacity violation, the ES rating should be at least equal to the maximum load demand and maximum energy supplied above the substation transformer rating in the five-year planning period i.e., the 100th percentile value. Instead of the worst-case rating, however, a representative rating is selected, based on the statistical analysis of the load demand profile.

The required MW capacity for ES is determined by subtracting the substation transformer emergency rating (75 MVA) from the percentile overload demand. The ES MW capacity obtained to meet a few percentile overloading conditions are shown in Table 2. Similarly, the MWh rating for ES is obtained using the percentile analysis results on MWh supplied above the transformer rating. The MWh ratings for ES, corresponding to several percentiles, are shown in Table 2. The ES capacity (both MW and $\mathrm{MWh}$ ) decreases significantly from the 100th-percentile to the 75th-percentile. When sizing ES for the 75th-percentile value, under the worst-case scenario, there is a 0.25 probability that the ES will be unable to mitigate a transformer overload. The 0.25 probability of not meeting the contingency requirement occurs when the transformer overloading occurs with a probability of 1 . To realistically estimate the risk for the five-year planning horizon, the probability of observing a transformer overload should be included in the risk calculation (Table 2). For the given circuit, in five years, an overloading under an $\mathrm{N}-1$ contingency scenario was recorded for a total of $641 \mathrm{~h}$. Thus, the probability of overloading under an 
$\mathrm{N}-1$ contingency scenario is equal to 0.0146 . If the ES is sized for the 75th-percentiles peak load, the N-1 contingency for the transformer will not be met with a probability $p_{\text {fail }}=0.25 \times 0.0146=36.5 \times 10^{-4}$, assuming that a failure in T1 is a certain event. The risk will further decrease when including the actual probability of observing a failure of the substation transformer.

Table 2. Percentile-based ES size (kW).

\begin{tabular}{cccc}
\hline \multirow{2}{*}{ Percentile } & \multicolumn{2}{c}{ Representative ES Size } & $\begin{array}{c}\text { Probability that N-1 Contingency not Met (Assuming } \\
\text { that the Fault Happens with Probability 1) }\end{array}$ \\
\cline { 2 - 3 } & MW & MWh & 0 \\
90 & 11.73 & 115.53 & $1.46 \times 10^{-4}$ \\
97 & 9.73 & 97.34 & $4.38 \times 10^{-4}$ \\
95 & 8.50 & 68.73 & $7.30 \times 10^{-4}$ \\
90 & 7.99 & 61.56 & $14.60 \times 10^{-4}$ \\
75 & 7.07 & 52.98 & $36.50 \times 10^{-4}$ \\
50 (median) & 5.74 & 30.28 & $73.00 \times 10^{-4}$ \\
\hline
\end{tabular}

In this study, the 75th-percetile value is selected for sizing the energy storage unit. Based on the analysis, the total ES system deployed in the feeder has a capacity of $6 \mathrm{MW} / 30 \mathrm{MWh}$. Note that a risk-averse deployment may select the ES size based on a 95th- or 97th-percentile calculation. However, the corresponding ES size may not be viable for deployment, given the exorbitant cost.

\subsubsection{Identify Energy Storage Location}

For N-1 contingency support, the ES system can be located anywhere, along either of the distribution feeders. Similarly, for frequency regulation application, ES can be deployed anywhere along the feeder and dispatched using the communication network in response to the frequency signal. However, the ES location is important for voltage management application. In order to mitigate the voltage variations due to PV variability, ES must be located close to the PV systems. Therefore, in this paper, ES is integrated and co-located at the 480-V level with each PV system. The ES circuit is supplied by a 1.2 MVA 34.5/0.48 kV service transformer. The topology of the secondary distribution circuit at the ES location is shown in Figure 10. The total ES capacity based on N-1 contingency analysis is $6 \mathrm{MW} / 30 \mathrm{MWh}$. This capacity is distributed at the following five PV locations. Since all of the PV systems are of the same capacity, we should ideally deploy a 1.2 MW/6 MWh ES system at each location. However, before deploying ES at each PV location, the largest capacity that can be integrated at the selected locations need to be calculated. This is called the ES accommodation capacity of the feeder for the given location.

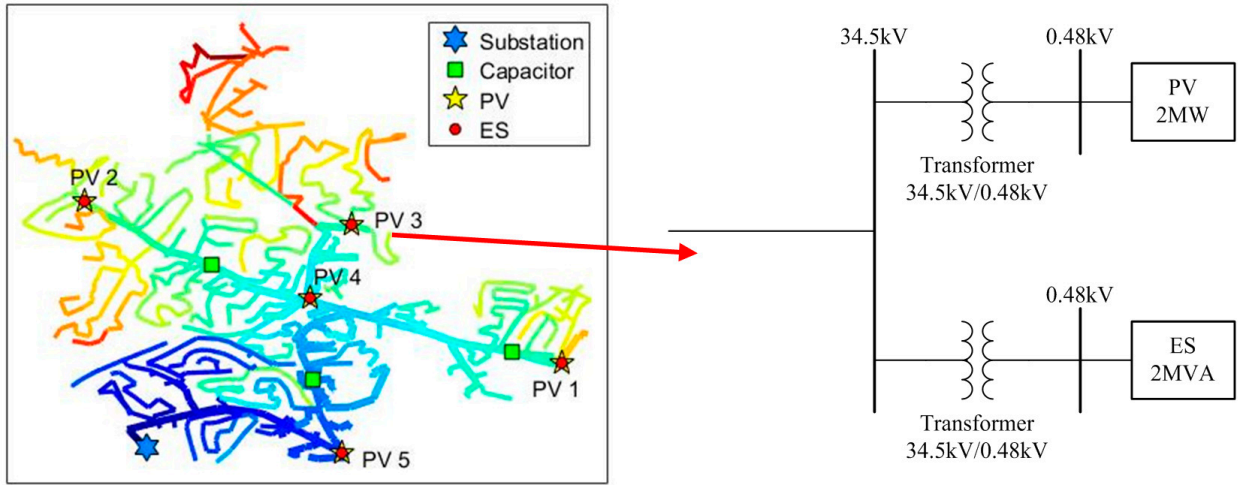

Figure 10. Topology of the secondary distribution circuit at the ES location. 


\subsubsection{Calculate Energy Storage Accommodation Limit}

ES may affect the normal operating conditions of the test feeder. Therefore, the largest ES capacity that can be installed at the selected location must be determined before integrating ES into the feeder. In this paper, the obtained ES capacity is referred to as the ES accommodation capacity. Since one of the stacked-benefits of ES is to provide a voltage management service, the voltage of the selected feeder must be kept within the acceptable limit, i.e., between 0.95 and 1.05 pu [33]. Hence, the size of the ES must be less than the largest ES capacity that can be installed without resulting in a steady-state voltage limit violation, overvoltage $(V>1.05 \mathrm{pu})$, or undervoltage $(V<0.95 \mathrm{pu})$, at the bus connected to the ES system (480-V level). In order to obtain the ES capacity limit, this paper determines the largest ES size resulting in an overvoltage (ES capacity limit for the overvoltage condition) and the largest ES size resulting in an undervoltage (ES capacity limit for the undervoltage condition), using a simulation approach performed in OpenDSS.

For the overvoltage case, the analysis aims at determining the largest generation without causing the overvoltage at the selected ES location. The simulation conditions assumed for the analysis are detailed as follows. The test feeder is assumed to be operating at the minimum load condition, thus resulting in a high voltage condition. The ES is set to discharge at its full rated output (acting as a generator) with a unity power factor, thus, causing the voltage to rise. The ES power rating is increased from 0 to $5 \mathrm{MVA}$, at an increment of $1 \mathrm{kVA}$. Since the ES is discharging, all of the PVs in the circuit are assumed to be not generating. The phase voltages at the ES location (480-V level) are recorded for each ES capacity (0-5 MVA). The ES accommodation capacity of the circuit at the selected ES location for the overvoltage is equal to the minimum ES rating, resulting in a phase voltage greater than 1.05.

A similar simulation approach is used to determine the largest ES capacity, resulting in an undervoltage violation $(\mathrm{V}<0.95 \mathrm{pu}$ ). The obtained ES capacity is referred to as the ES accommodation capacity for undervoltage limit violation. The simulation conditions and methodology are detailed as follows. For the worst-case scenario, the selected distribution circuit is assumed to be operating at the peak load condition and the ES is programmed to charge. The ES power rating is increased from 0 to $10 \mathrm{MVA}$, with an increment of $1 \mathrm{kVA}$. Since the ES is assumed to be charging, all of the PVs are assumed to be generating at their rated power with a unity power factor. The phase voltages at the ES location are recorded for each ES capacity (0-10 MVA). The ES capacity limit for the undervoltage condition is equal to the minimum ES rating, resulting in a phase voltage less than 0.95 pu at each ES location.

The phase voltages corresponding to the ES capacity for one of PV bus locations are shown in Figure 11. Based on the results, the overvoltage violation ( $\mathrm{V}>1.05 \mathrm{pu})$ is recorded when adding 3 MVA of ES, while the undervoltage violation $(\mathrm{V}<0.95 \mathrm{pu})$ is recorded when adding 9.1 MVA of ES. Thus, the ES accommodation capacity for the overvoltage condition is $3 \mathrm{MVA}$, and is 9.1 MVA for the undervoltage condition. Since the integration of ES must not cause either overvoltage or undervoltage limit violations in the circuit, the overall ES capacity limit of this circuit at the selected ES location is equal to 3 MVA (the lesser value). The same analysis is repeated for each PV location, and the ES accommodation capacities are obtained (please see Table 3). Based on the analysis, an ES system with a capacity of 1.2 MW / $6 \mathrm{MWh}$ can be deployed at each PV location, amounting to a total capacity of $6 \mathrm{MW} / 30 \mathrm{MWh}$. 


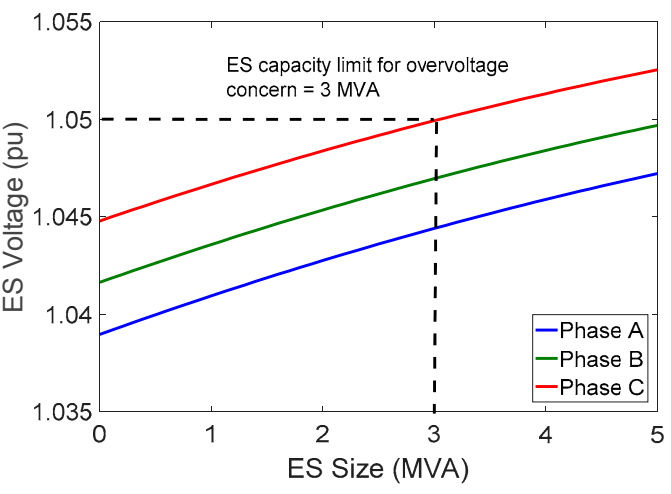

(a)

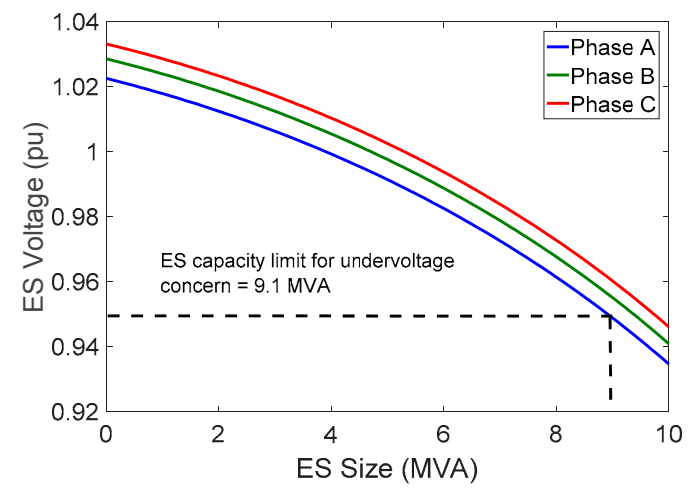

(b)

Figure 11. ES accommodation limit obtained for (a) overvoltage concern; (b) undervoltage concern.

Table 3. ES accommodation capacity at difference PV bus locations.

\begin{tabular}{ccc}
\hline \multirow{2}{*}{ PV Location } & \multicolumn{2}{c}{ ES Accommodation Capacity } \\
\cline { 2 - 3 } & Overvoltage Limit & Undervoltage Limit \\
\hline PV 1 & 3.4 & 9.3 \\
PV 2 & 3.3 & 9.5 \\
PV 3 & 3.0 & 9.1 \\
PV 4 & 3.7 & 9.7 \\
PV 5 & 3.1 & 10 \\
\hline
\end{tabular}

\subsubsection{Grid Impact Analysis}

The final task is to conduct a grid impact analysis to demonstrate that an ES application can provide the required stacked-benefits. First, the application of ES for meeting the N-1 contingency requirements is demonstrated. Since the ES size is selected based on the 75th percentile value of the overload demand and energy, the deployed ES will not be able to eliminate each possible case of overloading over the course of five years. However, we should expect a decrease in the overload demand and overloading duration after deploying the ES system. Next, the application of ES in meeting secondary grid benefits i.e., voltage management and frequency regulation, are discussed.

\section{Results and Discussion}

Based on the analysis presented in Section 3, five ES systems, each with a rating of $1.2 \mathrm{MW} / 6 \mathrm{MWh}$, are collocated with the PV systems in Feeder 1. The ES systems are programmed for 100\% commitment when the N-1 contingency is not met. For the rest of the time, ES is programmed to provide voltage management and frequency regulation services, each with $50 \%$ commitment. The utility of the above deployment method in providing the stacked-benefits is detailed in this section.

\subsection{Primary Application Scenario-N-1 Contingency Analysis}

The utility of deploying a 75th-percentile ES capacity, which corresponds to 6MW/30MWh, for meeting the $\mathrm{N}-1$ contingency requirement, is demonstrated in this section. The ES is programmed to discharge, to keep the substation load demand less than $75 \mathrm{MW}$. The ES begins charging at 2 am every day. Figure 12 shows the reduction in the overload demand recorded during the five-year planning horizon. It can be seen that the deployed ES can provide the contingency requirement for most of the time. However, at the 75th-percentile, the $\mathrm{N}-1$ contingency requirement is not met for a few hours, as shown in Figure 12. 


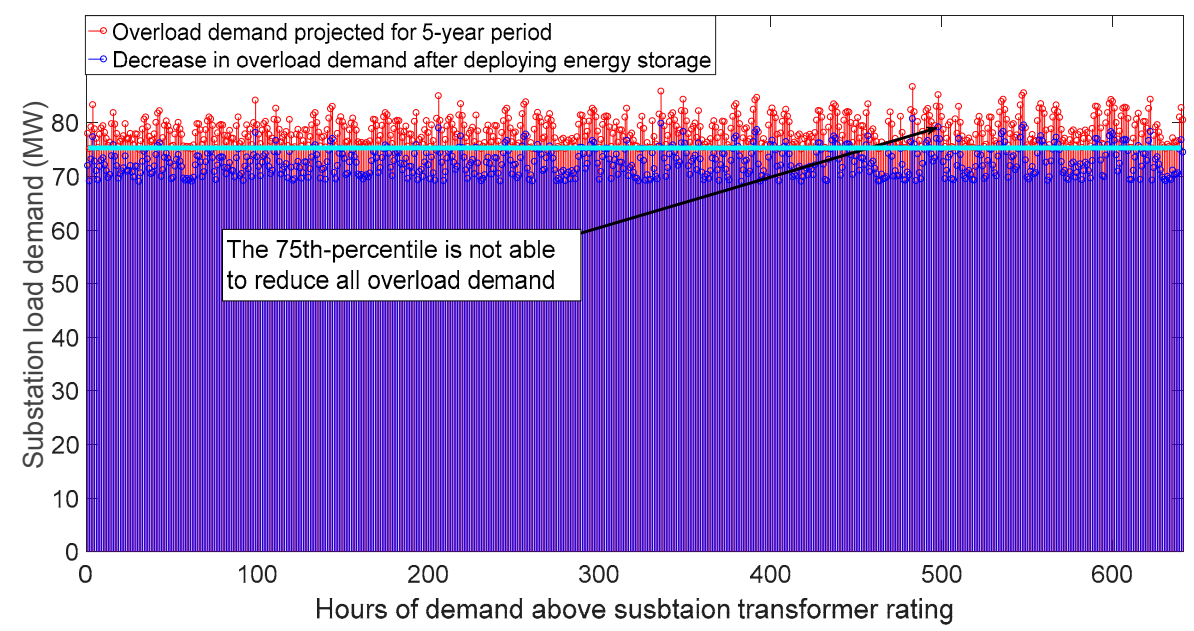

Figure 12. Application of energy storage in mitigating substation transformer overloading during an $\mathrm{N}-1$ contingency scenario.

Figure 13 compares the utility of the ES system for providing N-1 contingency support for the base year (Year 1) and Year 5. For this study, the load profiles for three consecutive days overloading the transformer under an N-1 contingency event are compared with and without an ES system. For the selected days in the base year (Year 1), the energy supplied above the transformer rating is $28.2 \mathrm{MWh}$, 29.05 MWh, and 28.6 MWh. In this case, the 30 MWh energy storage is successfully able to supply the load demand above the substation transformer rating. The analysis is repeated for the same days during Year-5 (see Figure 13b). On the selected days in Year-5, the energy supplied above the transformer rating is $55.36,77.29$ and $55.80 \mathrm{MWh}$. In this case, the $30 \mathrm{MWh}$ ES system is not able to successfully mitigate the overload concerns. As expected, the ES system sized for the 75th-percentile value is not able to supply the required energy demand for a few days during the planning duration.

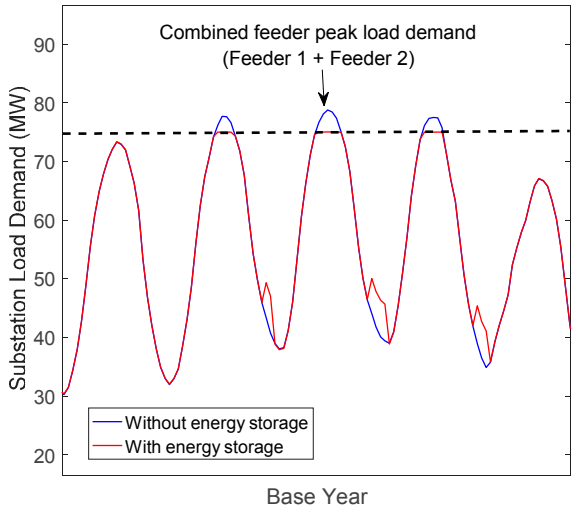

(a)

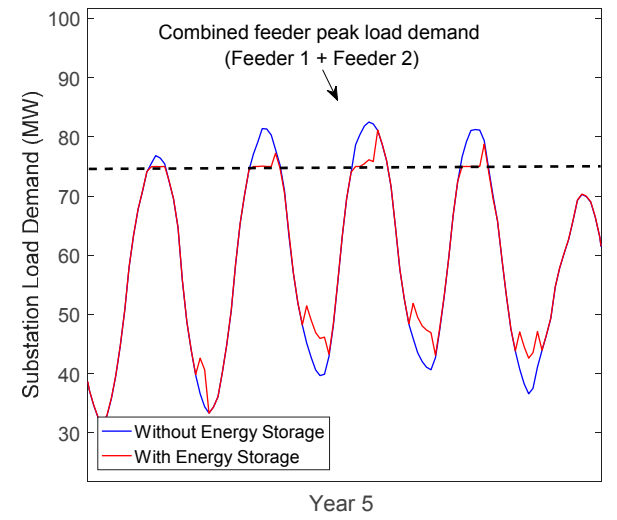

(b)

Figure 13. Application of energy storage in mitigating substation transformer overloading under an N-1 contingency scenario for a few selected days during: (a) Base year (Year-1), and (b) Year-5.

\subsection{Secondary Application Scenario-Stacked-Benefit Analysis}

For the stacked-benefit application, the ES system is deployed with $50 \%$ commitment for voltage management and $50 \%$ commitment for frequency regulation. The simulation is performed in OpenDSS for one hour, from 12 to $1 \mathrm{pm}$, with a time resolution of one second. Note that for the selected PV profile (see Figure 6a), the largest PV variability is observed around noon. In order to better understand the stacked benefits, four application scenarios are simulated: 
- Case 1-ES is not operating, i.e., the real and reactive power of ES are zero

- Case $2-50 \%$ of the ES capacity provides voltage management service due to PV variability only. ES is not providing any frequency regulation support.

- Case 3-ES simultaneously provides the following services:

Voltage management- $50 \%$ of the ES capacity provides voltage management service due to PV variability only

- Frequency regulation-50\% of the ES capacity provides frequency regulation service

- Case 4-ES simultaneously provides the following services:

- Voltage management- $50 \%$ of the ES capacity provides voltage management service due to both PV variability and the ES charge/discharge frequency regulation schedule

- Frequency regulation- $50 \%$ of the ES capacity is deployed to provide frequency regulation

The effectiveness of ES in providing voltage management and frequency regulation services is demonstrated using the ES real and reactive power output and the three-phase average voltage profiles recorded at the ES location. The profile for real power generated by all PV panels is presented in Figure 14. Recall that the total installed PV capacity is 5 MW. Figure 15a,b show the ES real and reactive power when ES is employed to provide frequency regulation and voltage management services, respectively.

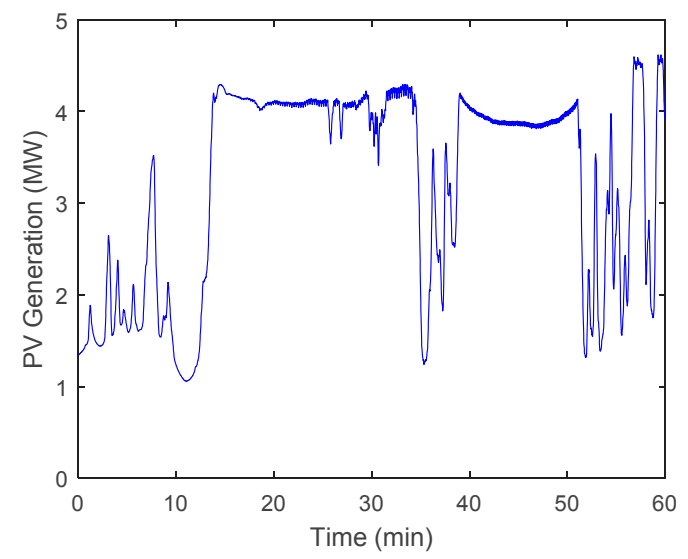

Figure 14. Total real power generated by PV panels from 12 p.m. to 1 p.m.

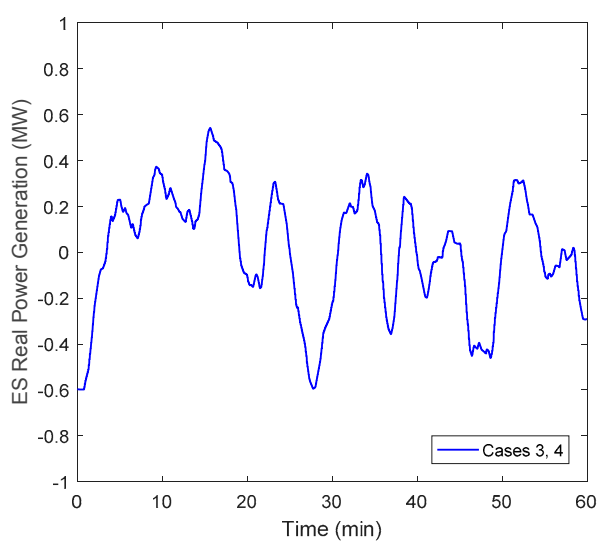

(a)

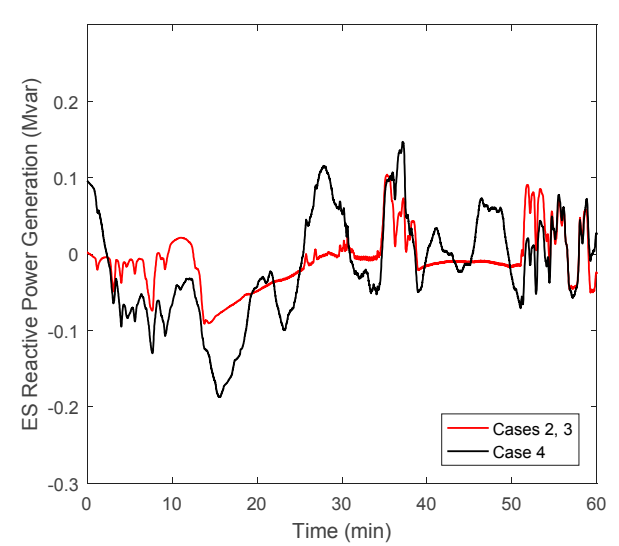

(b)

Figure 15. Application of ES for stacked-benefits: (a) Real power generated by one of the ES units for frequency regulation service; (b) Reactive power produced by ES when providing voltage management service. 
The voltage profiles for each case recorded at one of the ES locations (34.5-kV level) are shown in Figure 16. In Case 2, the voltage variability significantly reduces when ES is providing voltage management due to PV variability. However, the voltage variability significantly increases for Case 3. Recall that in Case 3,50\% of the ES capacity is deployed to provide frequency regulation services, while the remaining 50\% is committed to voltage management for voltage variability resulting only from PV systems. Due to frequency regulation services, ES systems also result in voltage variability, which needs to be accounted for during the voltage management. In Case 4, when ES is providing a voltage management service due to both PV variability and the ES charge/discharge frequency regulation schedule, the voltage variability decreases significantly.

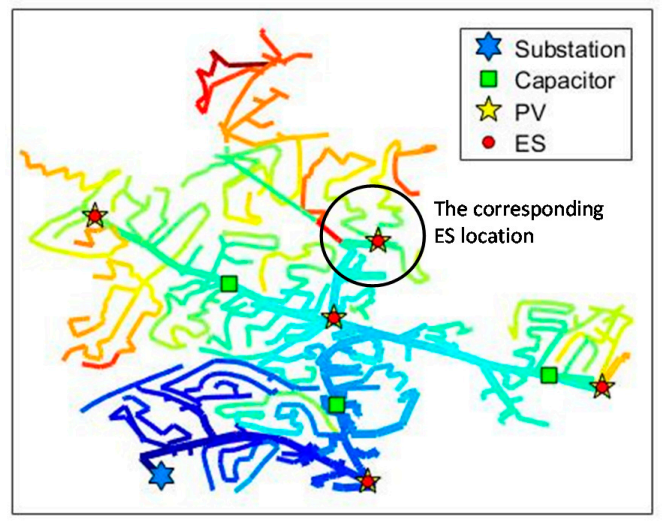

(a)

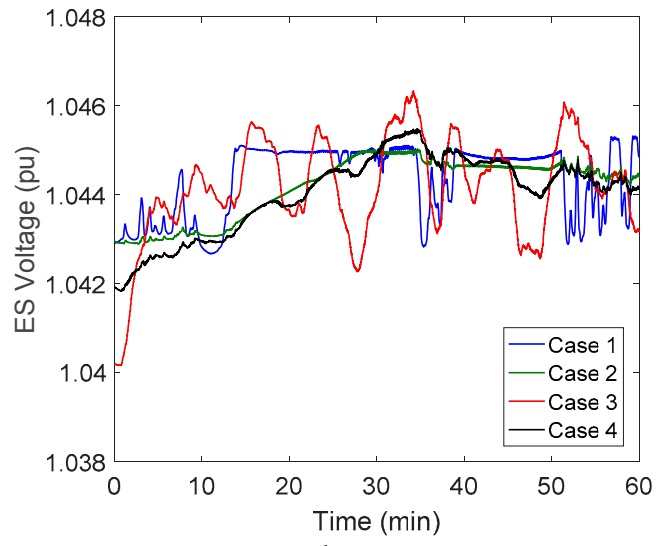

(b)

Figure 16. Voltage profiles at ES system location for different case studies: (a) the selected test distribution circuit and ES locations; (b) voltage profiles at one ES system location.

For the ease of comparison, the voltage variability is quantified using the voltage variability index (VVI) quantified using the standard deviation of the voltage variability recorded over the simulation period. The voltage variability is calculated as the difference between the present voltage and the voltage recorded during the previous time step. VVI is calculated using 1-min average data of voltage profiles. The VVI for each case is summarized in Table 4.

Table 4. Voltage Variability Index (VVI) for Various Cases.

\begin{tabular}{cccccc}
\hline VVI & PV 1 & PV 2 & PV 3 & PV 4 & PV 5 \\
\hline Case 1 & 8.11 & 7.72 & 9.15 & 7.58 & 5.56 \\
Case 2 & 1.39 & 1.46 & 1.38 & 1.48 & 1.84 \\
Case 3 & 10.83 & 10.37 & 11.96 & 10.20 & 7.81 \\
Case 4 & 1.91 & 1.87 & 2.64 & 1.86 & 2.16 \\
\hline
\end{tabular}

During the simulation period, a large variation in PV generation is recorded, as shown in Figure 14. When ES is not operating in Case 1, the fluctuation of PV generation results in significant voltage variability, as shown in Figure 16 and Table 4. After deploying ES for voltage management due to PV variability in Case 2, ES adjusts the reactive power to mitigate voltage variations. According to Table 4, the voltage variability decreases at each ES location. Thus, ES can successfully mitigate short-term voltage variations due to PV generation.

On employing ES for frequency regulation in addition to voltage management due to PV variability (Case 3), the real power of ES follows the frequency regulation schedule, as presented in Figure 15a. Therefore, ES is successfully able to charge and discharge to provide a frequency regulation service. However, Figure 16 shows that the voltage profile for this case fluctuates significantly, due to the variation of ES real power. From Table 4, the voltage variability significantly increases when ES is 
deployed for providing frequency regulation services. Therefore, ES should be deployed to manage voltage variability due to both PV and ES real power variations.

When ES is also used to provide voltage management due to the frequency regulation schedule in Case 4, Figure 15b shows that ES provides reactive power support based on both PV variability and the frequency regulation schedule. Hence, the voltage variability due to the frequency regulation schedule is reduced, as illustrated in Figure 16. From Table 4, for Case 4, the voltage variability decreases at each ES location.

\section{Conclusions}

This paper presents a planning framework for integrating energy storage systems into the distribution system. The objective is to deploy the ES system for providing stacked-benefits for the grid. We present a systematic approach, starting by identifying the ES application scenario for the feeder and then presenting an ES deployment framework. For the given feeder, depending upon the load demand data and current and future service requirements, primary and secondary applications for ES deployment are identified. For the selected feeder, ES is primarily deployed to provide the $\mathrm{N}-1$ contingency requirement for the feeder. For the stacked-benefit application, the ES system is deployed to provide voltage management and frequency regulation services. The presented ES deployment framework is based on the following four steps: (1) calculate ES system size based on primary application scenario; (2) determine optimal ES locations based on both primary and secondary application scenarios; (3) calculate the ES accommodation capacity at each potential ES location (4) deploy the ES system and develop a methodology for grid impact analysis to validate if the intended benefits are provided by the ES systems.

Furthermore, the paper also develops power control methods and demonstrates the stacked-benefit applications of deploying ES in simultaneously providing multiple services. For primary applications, $100 \%$ of the ES capacity would be deployed to provide the N-1 contingency requirement i.e., when one of the substation transformers is at fault, and the net load demand exceeds the other transformer's emergency rating. However, when the circuit is in a normal operating condition, $100 \%$ of the ES capacity would be used to provide other secondary services, such as voltage management and frequency regulation. In providing frequency regulation services, ES employs a real power control mode. In this control mode, ES charges and discharges in accordance with the frequency regulation schedule. The simulation results show that while ES can successfully provide frequency regulation by charging and discharging, the frequency regulation signal may suddenly ramp up or down, thus resulting in voltage variations. Therefore, along with the variability of PV generation, ES should also be employed to provide voltage management for voltage variations due to the frequency regulation schedule. In this paper, we develop ES control in order to provide reactive power support for the decreasing voltage variability resulting from sudden ramps in PV generation and in the frequency regulation schedule. The simulation results validate that ES systems are able to successfully mitigate the voltage variation concerns due to both the variability of PV generation and the frequency regulation schedule.

The proposed ES deployment framework is generic and can be easily adapted for other feeders, with a different set of primary and secondary application scenarios. The paper also outlines the characteristics of the modeling framework with the required dataset, thus presenting an important guideline for ES integration analysis. Finally, by demonstrating the application of ES in providing stacked grid benefits, this paper provides a successful use case for ES deployment in smart distribution systems.

It should be noted that rapid charging and discharging of the battery may deteriorate its overall lifetime. In this framework, to provide frequency regulation services, ES is generating/absorbing active power by rapidly charging/discharging every $2 \mathrm{~s}$. Usually, battery technologies are rated for several deep charge or discharge cycles, after which deterioration is expected. Fortunately, when participating in frequency regulation, the energy storage does not undergo deep charge or discharge every $2 \mathrm{~s}$. 
Instead, a few cycles of deep charge or discharge are observed over an entire day (Figure 15). Therefore, the framework may be feasible for existing battery systems if the benefit of providing frequency regulation offsets the cost of battery deterioration. However, an actual analysis on lifetime deterioration will depend upon the depth of charge/discharge in each cycle and the type of battery technology. In this paper, we do not model the battery lifetime deterioration due to charging/discharging-a potential topic for future research.

Author Contributions: Anamika Dubey, Pisitpol Chirapongsananurak, and Surya Santoso collaboratively developed the idea, the problem formulation, and the test framework. Anamika Dubey conducted the simulations, performed the data analysis, and wrote the paper; Pisitpol Chirapongsananurak helped with the simulations, analysis, and paper writing; Surya Santoso helped with the analysis and the interpretation of the results.

Conflicts of Interest: The authors declare no conflict of interest.

\section{References}

1. Ipakchi, A.; Albuyeh, F. Grid of the future. IEEE Power Energy Mag. 2009, 7, 52-62. [CrossRef]

2. Peças Lopesa, J.A.; Hatziargyrioub, N.; Mutalec, J.; Djapicc, P.; Jenkinsc, N. Integrating distributed generation into electric power systems: A review of drivers, challenges and opportunities. Electr. Power Syst. Res. (Elsevier) 2007, 77, 1189-1203. [CrossRef]

3. Executive Office of the President. National Science and Technology Council. In A Policy Framework for the 21st Century Grid: A Progress Report. Available online: https:/ /obamawhitehouse.archives.gov/sites/default/ files/microsites/ostp/2013_nstc_grid.pdf (accessed on 24th February 2017).

4. U.S. Department of Energy. The American Recovery and Reinvestment Act Smart Grid Highlights. Available online: https:/ /energy.gov/sites/prod/files/2014/12/f19/SGIG-SGDP-Highlights-October2014. pdf (accessed on 24th February 2017).

5. U.S. Department of Energy, Office of Energy Efficiency \& Renewable Energy. Vehicle Technologies Program Multi-Year Program Plan (2011-2015). Available online: http:/ /arge.stanford.edu/courses/2011/ph240/ zhong1/docs/vt_mypp_2011-2015.pdf (accessed on 24th February 2017).

6. Katiraei, F.; Aguero, J.R. Solar PV integration challenges. IEEE Power Energy Mag. 2011, 9, 62-71. [CrossRef]

7. Erdman, W. Secondary Network Distribution Systems Background and Issues Related to the Interconnection of Distributed Resources; National Renewable Energy Laboratory: Golden, CO, USA, 2005.

8. Dubey, A.; Santoso, S. Electric vehicle charging on residential distribution systems: Impacts and mitigations. IEEE Access 2015, 3, 1871-1893. [CrossRef]

9. Brown, R.E. Impact of smart grid on distribution system design. In Proceedings of the IEEE Power and Energy Society General Meeting-Conversion and Delivery of Electrical Energy in the 21st Century, Pittsburgh, PA, USA, 20-24 July 2008.

10. California Energy Commission and KEMA Inc. Research Evaluation of Wind Generation, Solar Generation, and Storage Impact on the California Grid. CEC PIER Final Project Report, CEC-500-2010. 2010. Available online: http:/ / www.energy.ca.gov/2010publications/CEC-500-2010-010/CEC-500-2010-010.PDF (accessed on 24th February 2017).

11. Rittershausen, J.; McDonagh, M. Moving Energy Storage from Concept to Reality; Southern California Edison: Rosemead, CA, USA, 2011.

12. Eyer, J.; Corey, G. Energy Storage for the Electricity Grid: Benefits and Market Potential Assessment Guide; SAND2010-0815; Sandia National Laboratories: Albuquerqu, NM, USA, 2010.

13. Abrams, A.; Fioravanti, R.; Harrison, J.; Katzenstein, W.; Kleinberg, M.; Lahiri, S.; Vartanian, C. Energy Storage Cost Effectiveness Methodology and Preliminary Results; California Energy Commission: Sacramento, CA, USA, 2013.

14. Chen, C.; Duan, S.; Cai, T.; Liu, B. Optimal allocation and economic analysis of energy storage system in microgrids. IEEE Trans. Power Electron. 2011, 26, 2762-2773. [CrossRef]

15. Xu, X.; Bishop, M.; Oikarinen, D.G.; Hao, C. Application and modeling of battery energy storage in power systems. CSEE J. Power Energy Syst. 2016, 2, 82-90. [CrossRef]

16. Atwa, Y.M.; El-Saadany, E.F. Optimal allocation of ESS in distribution systems with a high penetration of wind energy. IEEE Trans. Power Syst. 2010, 25, 1815-1822. [CrossRef] 
17. Barton, J.P.; Infield, D.G. Energy storage and its use with intermittent renewable energy. IEEE Trans. Energy Convers. 2004, 19, 441-448. [CrossRef]

18. Hill, C.A.; Such, M.C.; Chen, D.; Gonzalez, J.; Grady, W.M. Battery energy storage for enabling integration of distributed solar power generation. IEEE Trans. Smart Grid 2012, 3, 850-857. [CrossRef]

19. Wang, G.; Ciobotaru, M.; Agelidis, V.G. Power management for improved dispatch of utility-scale PV plants. IEEE Trans. Power Syst. 2016, 31, 2297-2306. [CrossRef]

20. Bass, R.B.; Carr, J.; Aguilar, J.; Whitener, K. Determining the power and energy capacities of a battery energy storage system to accommodate high photovoltaic penetration on a distribution feeder. IEEE Power Energy Technol. Syst. J. 2016, 3, 119-127. [CrossRef]

21. Yang, Y.; Li, H.; Aichhorn, A.; Zheng, J.; Greenleaf, M. Sizing strategy of distributed battery storage system with high penetration of photovoltaic for voltage regulation and peak load shaving. IEEE Trans. Smart Grid 2014, 5, 982-991. [CrossRef]

22. Gantz, J.M.; Amin, S.M.; Giacomoni, A.M. Optimal mix and placement of energy storage systems in power distribution networks for reduced outage costs. In Proceedings of the IEEE Energy Conversion Congress and Exposition, Raleigh, NC, USA, 15-20 September 2012; pp. 2447-2453.

23. Wang, Y.; Tan, K.T.; Peng, X.Y.; So, P.L. Coordinated control of distributed energy-storage systems for voltage regulation in distribution networks. IEEE Trans. Power Deliv. 2016, 31, 1132-1141. [CrossRef]

24. Han, Y.; Young, P.M.; Jain, A.; Zimmerle, D. Robust control for microgrid frequency deviation reduction with attached storage system. IEEE Trans. Smart Grid 2015, 6, 557-565. [CrossRef]

25. Kim, Y.; Del-Rosario-Calaf, G.; Norford, L.K. Analysis and experimental implementation of grid frequency regulation using behind-the-meter batteries compensating for fast load demand variations. IEEE Trans. Power Syst. 2017, 32, 484-498. [CrossRef]

26. U.S. Department of Energy. Grid Energy Storage. Available online: http://www.sandia.gov/ess/docs/other/ Grid_Energy_Storage_Dec_2013.pdf (accessed on 24th February 2017).

27. Bender, D.; Byrne, R.; Borneo, D. ARRA Energy Storage Demonstration Projects: Lessons Learned and Recommendations; SAND2015-5242; Sandia National Laboratories: Albuquerque, NM, USA, 2015.

28. Dugan, R.C. The Open Distribution System Simulator ${ }^{\mathrm{TM}}$ (OpenDSS), Reference Guide; Electric Power Research Institute, Inc.: Palo Alto, CA, USA, 2012.

29. Smart Grid Resource Center. Electric Power Research Institute. Available online: http://smartgrid.epri. com/SimulationTool.aspx (accessed on 24th February 2017).

30. Reno, M.J.; Coogan, K. Grid Integrated Distributed PV (GridPV) Version 2; SAND2014-20141; Sandia National Laboratories: Albuquerque, NM, USA, 2014.

31. Chirapongsananurak, P.; Dubey, A.; Jothibasu, S.; Santoso, S.; Maitra, A. Stacked benefit applications of energy storage systems in distribution circuits. In Proceedings of the IEEE PES General Meeting, Chicago, IL, USA, 16-20 July 2017. accepted.

32. Ancillary Services. PJM. Available online: http://www.pjm.com/markets-and-operations/ancillaryservices.aspx (accessed on 24th February 2017).

33. American National Standard for Electric Power Systems and Equipment-Voltage Ratings (60 Hz); ANSI C84.1-2011; National Electrical Manufacturers Association: Rosslyn, VA, USA, 2011.

(C) 2017 by the authors. Licensee MDPI, Basel, Switzerland. This article is an open access article distributed under the terms and conditions of the Creative Commons Attribution (CC BY) license (http:/ / creativecommons.org/licenses/by/4.0/). 